\title{
Automated Identification and Quantitation of Four Patterns of Electrocortical Activity in the Near-Term Fetal Lamb
}

\author{
MARY ELLEN MCNERNEY AND HAZEL H. SZETO \\ Department of Pharmacology, Cornell University Medical College, New York, New York 10021
}

\begin{abstract}
Most previous efforts to characterize electrocortical states in the unanesthetized fetal lamb have relied on descriptive measures of amplitude and frequency; those that used quantitative parameters did not draw upon data from a cohort of subjects for contiguous time segments. We have used Fourier analysis to quantitate amplitude and frequency characteristics of records of $2-3 \mathrm{~h}$ for a group of fetuses. Four electrocortical states, including two types of transitional activity, have been identified and quantitated. Additionally, we have developed a method that automates the assignment of data to these four electrocortical states. This has enabled us to monitor transitions among states and indicates that epochs that appear homogeneous by descriptive measures may actually encompass several patterns of electrocortical activity. (Pediatr Res 28: 106-110, 1990)
\end{abstract}

\section{Abbreviations}

ECoG, electrocorticogram

HVSA, high-voltage, slow activity

LVFA, low-voltage, fast activity

FFT, Fast Fourier Transform

The fetal lamb begins to exhibit cyclic alternations among discrete patterns of electrocortical activity at approximately 115 $\mathrm{d}($ term $=145 \mathrm{~d})$. Two predominant patterns, or states, have been identified. These are variously termed HVSA and LVFA (1), or simply, high-voltage electrocortical activity and low-voltage electrocortical activity (2). The properties of each state may be defined by quantitation of both amplitude and frequency components of the electrocortical waveform. Initial attempts to characterize the developing ECoG relied upon descriptions of its amplitude as obtained from visual analysis of polygraphic records. There were two problems with this approach. First, visual analysis is hindered by subjectivity. Second, records were generally transcribed at low polygraphic paper speeds; this does not permit the assessment of component frequencies. More recently, attempts have been made to quantitate frequency characteristics of the ECoG in the fetal lamb; these have focused on the two preponderant states. The FFT has been used in our own (3) and other $(4,5)$ laboratories for this purpose; this algorithm permits

Received January 25, 1990; accepted April 10, 1990.

Correspondence and reprint requests: Hazel H. Szeto, M.D., Ph.D., Department of Pharmacology, Cornell University Medical College, 1300 York Avenue, New York, NY 10021

Supported in part by the National Institute on Drug Abuse (DA02475-09). M.E.M. is a recipient of a predoctoral fellowship from the Pharmaceutical Manufacturers' Association Foundation. H.H.S. is the recipient of a Research Scientist Development Award from the National Institute on Drug Abuse (K02-DA0010006), ADAMHA. the quantitation of the contribution to amplitude of each constituent frequency in a bioelectric signal.

Only the report from our laboratory has quantitated the spectral characteristics for a cohort of fetuses, however (3). Technical restrictions at that time on data sampling abilities limited analysis to a small number of temporally isolated segments of the ECoG signal, which were selected by preliminary visual analysis. We have recently developed the capability to perform continuous off-line FFT for records of any length; the method is limited only by data storage capacity. Additionally, we have devised a method for automation of electrocortical state assignment that is based on the amplitude and frequency information derived from the FFT. We have examined continuous data segments of $2 \mathrm{~h}$ or longer. This has enabled us to produce detailed analyses of the frequency characteristics of HVSA and LVFA, as well as those of transitional epochs. Moreover, it has enabled us to examine the cycling from one state to another.

Electrocortical states, both preponderant and transitional, are identified and characterized as a continuum whose extremes are HVSA and LVFA. We propose that signals whose amplitude and frequency properties are intermediate between those of HVSA and LVFA are broadly divided into two additional states. We have found that the latter are observed within epochs identified visually as HVSA and LVFA, as well as during brief periods of transition between HVSA and LVFA.

\section{MATERIALS AND METHODS}

Animal preparation. Seven fetal lambs were surgically instrumented for chronic intrauterine recording of electrocortical activity between 121 and $125 \mathrm{~d}$ of gestation, in accord with guidelines approved by the institution for the care and use of the animals. Details of the surgical procedure have been described previously (6). Briefly, four stainless steel screws (size 0-80) were implanted in the left and right parietal bones (approximately 8 and $25 \mathrm{~mm}$ distal to the coronal suture, and $15 \mathrm{~mm}$ lateral to the sagittal suture) for recording ECoG activity. Leads were tunnelled s.c. to the maternal flank and stored in a pouch.

Recording procedures. Ewes were allowed at least $72 \mathrm{~h}$ after surgery before commencement of fetal monitoring. The range of gestational ages studied is $124-137 \mathrm{~d}$; this span represents recordings obtained $3-13$ d postoperatively. Polygraphic recordings were carried out with the ewe standing or lying quietly in a cart. All recordings, which were $2-3 \mathrm{~h}$ in length, were obtained at the same time of day (0900 through $1200 \mathrm{~h}$ ). The ewe had access to food and water throughout the recording period.

Data acquisition and processing. The ECoG was recorded on a model $2800 \mathrm{~S}$ (Gould Corp., Cleveland, $\mathrm{OH}$ ) analogue recorder after amplification to about $250 \mathrm{mV}$ with a bandpass filter of 1$100 \mathrm{~Hz}$. The amplified, filtered analogue signal was recorded concurrently onto FM tape (XR-310; TEAC Corp., Montebello, CA) for storage and off-line analysis.

Power spectral analysis. Analogue-to-digital conversion was 
accomplished with a board (DT-2801A, Data Translation, Waltham, MA) resident in an AT microcomputer (IBM Instruments, Inc., Danbury, CT). Data were continuously digitized off-line at a rate of $256 \mathrm{~Hz}$, and stored in the microcomputer in binary format.

Raw data were first corrected for the presence of a linear trend by subtracting from each data point the corresponding value of the best-fit line estimated using all data points. The presence of such a trend, if uncorrected, introduces artifactual power in the low-frequency components $(7,8)$. After removal of the linear trend, a Hamming window was applied. In the absence of a suitable window, the FFT of a digitized series-such as the series that results from our analog-to-digital conversion-would contain large artifacts in many frequencies of the spectrum $(7,8)$. FFT were performed on consecutive series of 1024 detrended, windowed data points $(4 \mathrm{~s})$. The resolution of the FFT was $1 / 4$ $\mathrm{Hz}$. The maximum detectable frequency was designated to be 64 $\mathrm{Hz}$, although data for frequencies $>32 \mathrm{~Hz}$ were discarded.

Five power spectra thus obtained were averaged to produce a single mean power spectrum, or run. (Averaging of spectra is necessary, as the error associated with each point in a single power spectrum is $100 \%$; the mean of $n$ runs of data produces an error term that is reduced to $1 /(n-1)^{1 / 2}(7)$.) Mean power spectra were written to a file.

Simultaneously, 50 and $90 \%$ spectral edges were calculated; these are the frequencies below which 50 and $90 \%$ of the power in a run reside. Spectral edges were also written to a file.

Subsequently, a number of spectral parameters were calculated for each run. These included total power and percent power in several EEG bandwidths, including those that are well-established markers in the adult: $1-4 \mathrm{~Hz}$ (delta), $5-7 \mathrm{~Hz}$ (theta), 8-10 $\mathrm{Hz}, 8-13 \mathrm{~Hz}$ (alpha), $11-14 \mathrm{~Hz}$ and $15-32 \mathrm{~Hz}$ (beta).

Spectral parameter data from individual fetuses were pooled within a given electrocortical state and mean spectral parameters for each state were derived for the group. These were initially evaluated for statistically significant differences between groups with the Kolmogorov-Smirnov test for distributions (9). When significant differences were observed, the nonparametric MannWhitney test $(9,10)$ was used to identify the source of the deviation. Both Kolmogorov-Smirnov and Mann-Whitney tests were implemented using the software package Systat V.4.0 (Systat, Inc., Evanston, IL).

The incidence of each state was calculated as:

$$
\text { Incidence }=100 \times \frac{\text { Number of runs in state } \mathrm{X}}{\text { Total number of runs in record }}
$$

Incidence data are reported for the group.

\section{RESULTS}

A total of 12 recordings were obtained from seven fetal lambs. Fetal blood gases and $\mathrm{pH}$ were within normal limits on the day of the study.

Figures 1, 2, and 3, as well as Table 2, are derived from a single fetus; they are included to illustrate, in detail, some of the preliminary results.

We have previously found that the $90 \%$ spectral edge is an excellent index of electrocortical cycling in the fetal lamb (11). Time series plots of this index were generated for each fetal record. A representative polygraphic record, as well as the corresponding spectral edge time series, are shown in Figure 1 (top and center panels).

When scrutinized in conjunction, the time series plot of the $90 \%$ spectral edge and the other spectral parameters listed above provide a guide to the range of spectral parameters to be found in stable episodes of HVSA and LVFA. We chose three parameters on which to base the automation of state assignments: total power, percent power in the delta $(1-4 \mathrm{~Hz})$ bandwidth, and percent power in the beta $(15-32 \mathrm{~Hz})$ bandwidth. The distinction of HVSA and LVFA may be accomplished with any one of these
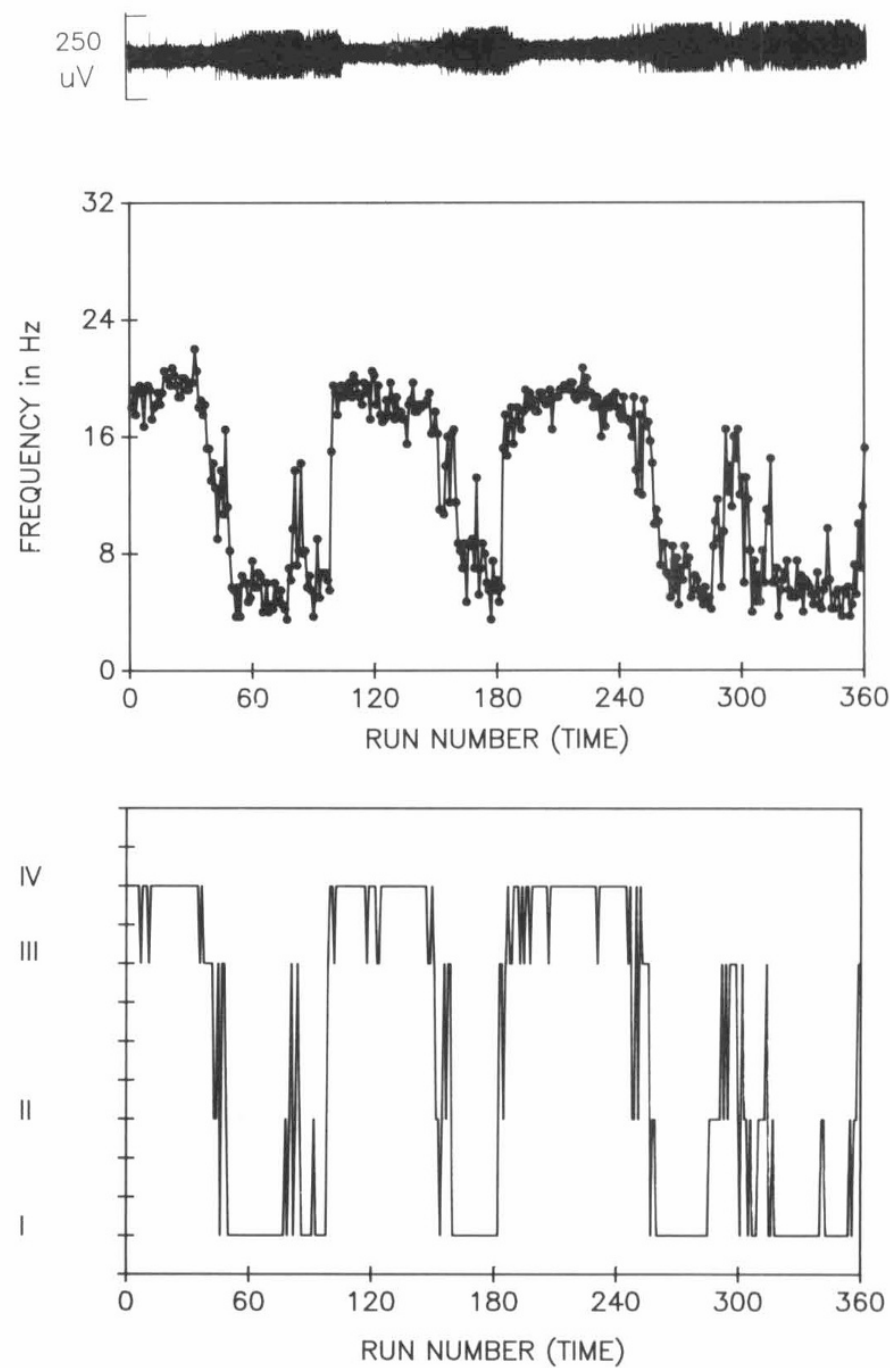

Fig. 1. Top panel: 120-min polygraphic recording of the ECoG of a fetal lamb (gestational age $128 \mathrm{~d}$ ). Full scale is approximately $250 \mu \mathrm{V}$. Center panel: time series plot of the $90 \%$ spectral edge for the interval depicted by the polygraphic trace. Bottom panel: time series plot of the state-to-state transitions for this interval.

criteria. Additionally, scrutiny of these parameters revealed that, taken together, it was possible to distinguish different types of transitional waveform; however, these could not be discriminated solely on the basis of any single spectral parameter, whether derived from amplitude (i.e. power) or frequency. Ultimately, it was found that automated state assignment for all electrocortical states could be accomplished by specification of numerical criteria for two of three parameters: 1) total power within a given run, 2) percent power in the 1-4 $\mathrm{Hz}$ bandwidth, and 3) percent power in the $15-32 \mathrm{~Hz}$ bandwidth. Spectral parameters from transitional signals, in particular, could be categorized into two clusters when parameters 2 and 3 were considered in conjunction. We found that, in addition to the two preponderant electrocortical states, it is useful to consider the existence of two transitional states.

Figures $2 A$ and $B$ depict two-dimensional histograms that illustrate the clustering of spectral parameters according to electrocortical state for a single fetus. Figure $2 A$ depicts total power versus percent delta power; the criterion for total power that distinguishes HVSA from a transitional state with similar percent delta power characteristics is identified as 18750 . Similarly, in Figure $2 B$ (which illustrates percent delta power versus percent beta power), it is possible to discern two major types of activity, which correspond to the two preponderant states, as well as intermediate patterns of activity, which we have broadly cate- 
A

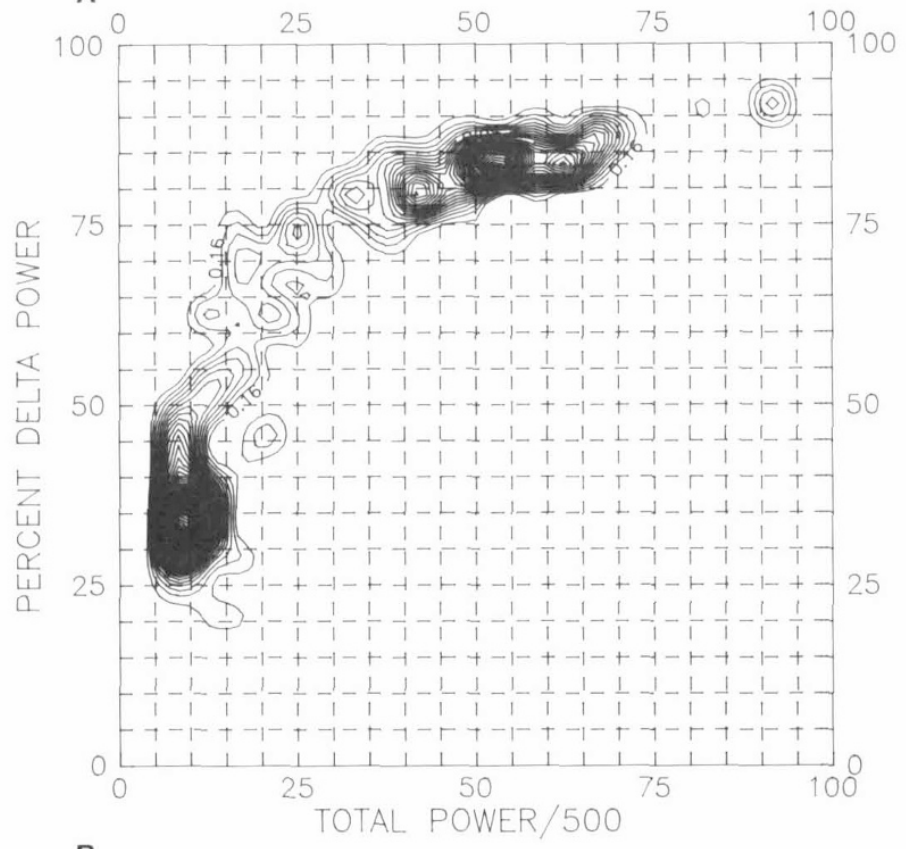

B

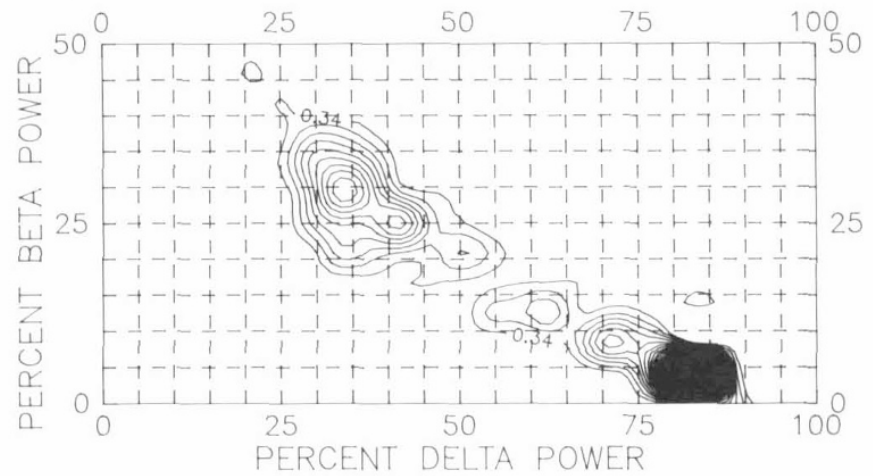

Fig. 2. Two-dimensional histograms (relative frequency of occurrence is reflected in the intensity of shading). $2 A$, total power $v s$ percent power in the delta $(1-4 \mathrm{~Hz})$ waveband. $x$ axis: total power/500. $y$ axis: percent power in delta. $2 \mathrm{~B}$. Percent power in the delta waveband vs percent power in the beta $(15-32 \mathrm{~Hz})$ waveband. $x$ axis: percent power in delta. y axis: percent power in beta. Solid lines in each figure indicate criteria for state assignment.

gorized into two intermediate states. Careful visual scrutiny of the raw data for every fetal record enabled an estimate of the range of values for each parameter in each of the four states. Automated assignment of runs to a given state was accomplished by establishment of criteria (i.e. numerical cutoffs for each of the three parameters listed above). The criteria used for this fetus are: state I (HVSA); percent power in $1-4 \mathrm{~Hz}$ is $>67.5 \%$ and total power is >18 750; state II (transitional state "a"), percent power in $1-4 \mathrm{~Hz}$ is $>67.5 \%$ and total power is $<18750$; state III (transitional state "b"), percent power in $1-4 \mathrm{~Hz}$ is $<67.5 \%$ and percent power in $15-32 \mathrm{~Hz}$ is $<20 \%$; and state IV (LVFA), percent power in $1-4 \mathrm{~Hz}$ is $<67.5 \%$ and percent power in $15-$ $32 \mathrm{~Hz}$ is $>20 \%$.

After the assignment of all runs in a fetal record to electrocortical states by these criteria, the states were compared to determine whether they differed significantly. The KolmogorovSmirnov Test was used for the data from individual records to compare states I and II, states II and III, and states III and IV for each of three spectral parameters: total power, percent power in 1-4 Hz, and percent power in $15-32 \mathrm{~Hz}$. Cutoff criteria for an individual fetal record were judged to be adequate if $a$ ) all comparisons were statistically significant $(p<0.05)$ and $b)$ run-
A

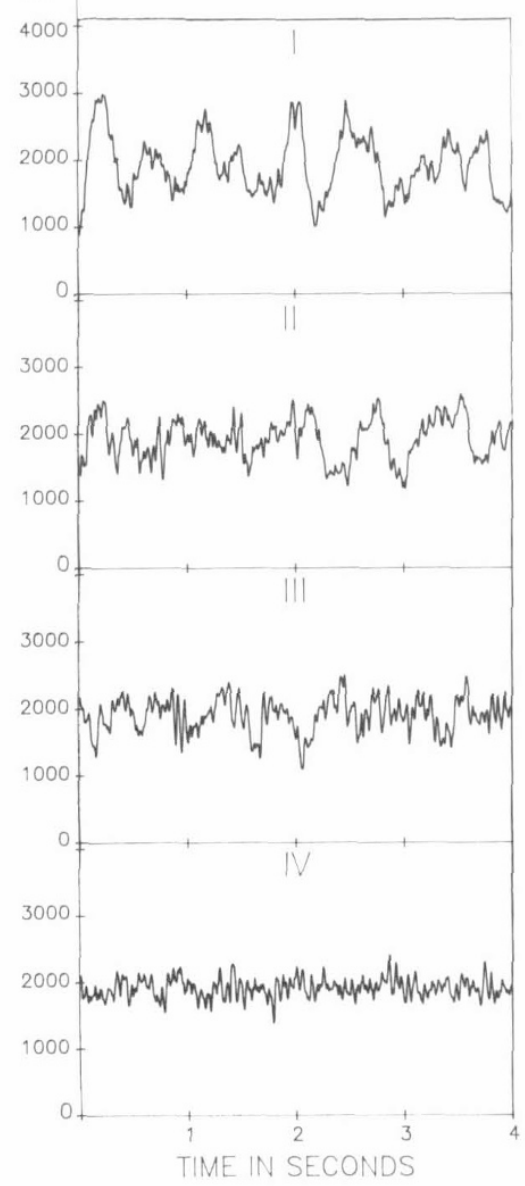

B

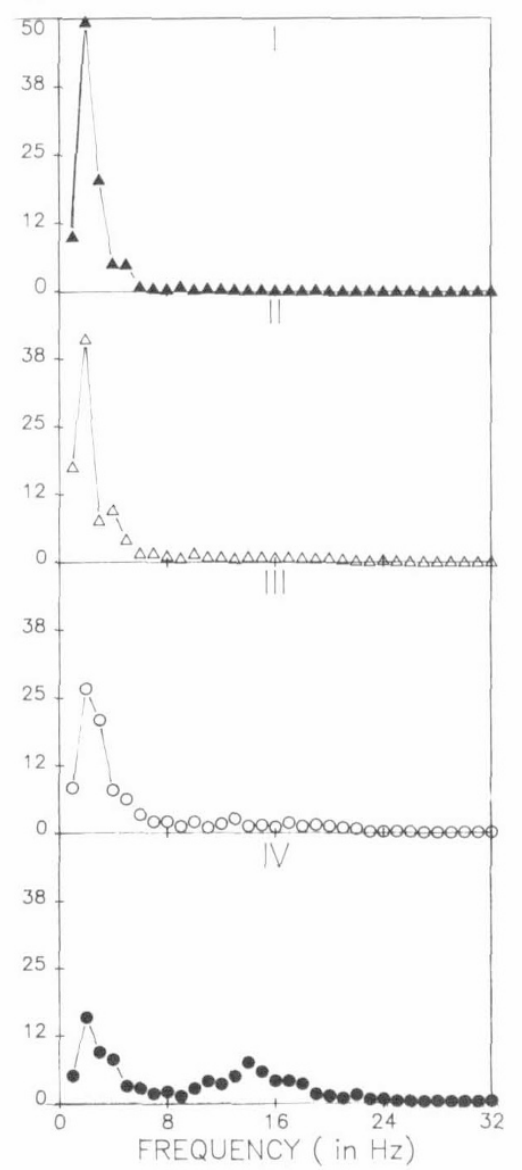


hampered by the inability to process more than $4 \mathrm{~s}$ of contiguous data (3). We have developed the capability for continuous data digitization in an inexpensive, microcomputer-based system. This permitted us to subject 12 2- to 3-h segments of ECoG signal from seven late-term fetal lambs to Fourier analysis. This type of extensive, quantitative analysis of developing electrocortical activity has not been previously reported. Furthermore, we have automated the assignment of data segments to electrocortical states; this obviates the subjectivity that is associated with visual assignment.

This approach has enabled us to identify and characterize four electrocortical states in the range of gestational ages studied (124137 d). Previous reports by other investigators who applied Fourier analysis to the ECoG of the fetal lamb $(4,5)$ have been limited to descriptions of the two preponderant states (HVSA and LVFA) and have not detailed results for more than one fetus. The four states that we have described represent a continuum in terms of amplitude and frequency. HVSA was designated state I; approximately $85 \%$ of total power resides in the delta band. Total power in this state is the greatest of the four states. The major feature that distinguishes HVSA from state II is the dramatic decline in total power in the latter; typically, it is only $33-40 \%$ of that in state I. Additionally, the power in delta is less than that observed in HVSA and the power in remaining bands is greater than that seen in HVSA.

The trends illustrated in state II are extended in state III: there is further reduction in total power $(\sim 20-25 \%$ that seen in state I), further erosion of power in delta frequencies $(\sim 63 \%)$, and enhancement of power in faster frequencies.

LVFA, designated state IV, has the least total power of the four states; typically, this is $\sim 10 \%$ of that observed in state I. Only $42 \%$ of total power resides in the delta band and there is a substantial fraction $(25 \%)$ observed in beta frequencies.

Data derived for $90 \%$ spectral edge for the four electrocortical states exhibit a trend that is consistent with the frequency continuum observed. The value for state I was lowest, that for state IV the greatest, and those for states II and III intermediate.

The incidences of states I and IV obtained by automated analysis ( 31 and $46 \%$, respectively) are somewhat lower than we have previously reported for HVSA and LVFA (38 and 60\%, respectively) by visual analysis (6). However, when taken together, the total incidence of states I and II (39\%); and states III and IV (61\%) are virtually identical to our earlier findings. Clearly, the automated method is capable of discriminating spectral parameters that cannot be distinguished visually, although inspection of prototypic data (Fig. 3 and Table 2) reveals that the differences among states I-IV are not particularly subtle.

States II and III were not exclusively observed during transitional epochs between HVSA and LVFA; they also occur within intervals of states I and IV. It is not surprising that analysis of state-to-state transitions reveals that epochs identified visually as states I and IV are not as homogeneous as they appear. Rather, there are routine excursions between states I and II and between states III and IV. Furthermore, transitional epochs are not sharply defined entities that occur between episodes of states I and IV; in fact, they are most frequently interspersed with activity that is quantitatively identified as state I or IV at the onset or termination of one of these preponderant states. The overall impression illustrated in Figure 1, bottom panel, is one of tem- porary vacillation between states before stabilization in a new preponderant state.

The quantitation of frequency and amplitude characteristics for states II and III is important for three reasons. First, there have been no attempts to quantitate the frequency and amplitude characteristics of transitional electrocortical activity in the fetus. Second, we report that waveforms of intermediate amplitude and frequency are consistently found within longer epochs of HVSA and LVFA; when records were visually analyzed, these were overlooked. Finally, it is well known that a number of pharmacologic and pathophysiologic perturbations in utero induce electrocortical states of intermediate amplitude and (previously) indeterminate frequency. The characterization of states II and III enables comparisons between physiologic conditions and those established by such perturbations.

The significance of this work to the question of fetal sleep staging cannot be determined definitively without concomitant polygraphic records of postural muscle tone and eye movements (12); these recordings were not available for these studies. Nonetheless, our previous findings allow us to make a number of inferences regarding states I and IV. State I (HVSA) seems to represent the electrocortical component of the deeper planes of quiet sleep. State IV probably corresponds to the presence of arousal and/or rapid-eye-movement sleep; these two behavioral states are both associated with LVFA, and can only be differentially distinguished by the presence of postural muscle tone and rapid eye movements. Further investigation will attempt to corroborate these inferences, as well as identify the sleep stages that correspond to states II and III in the normal developing fetus.

Acknowledgments. The authors thank Dr. Eugene Dwyer for his assistance in software development. Additionally, we are indebted to Dr. Antonio Sastre and especially to Dr. Paul Blank for our education in the rigors of Fourier analysis.

\section{REFERENCES}

1. Clapp JF, Szeto HH, Abrams R, Larrow R, Mann LI 1980 Physiologic variability and fetal electrocortical activity. Am J Obstet Gynecol 136:10451051

2. Dawes GS, Fox HE, Leduc BM, Liggins GC, Richards RT 1972 Respiratory movements and rapid eye movement sleep in the fetal lamb. J Physiol (Lond) 220:119-143

3. Szeto HH, Vo TD, Dwyer G, Dogramajian ME, Cox M, Senger G 1985 The ontogeny of fetal lamb electrocortical activity: a power spectral analysis. Am J Obstet Gynecol 153:462-468

4. Clewlow F, Dawes GS, Johnston BM, Walker DW 1983 Changes in breathing, electrocortical and muscle activity in unanaesthetized fetal lambs with age. J Physiol (Lond) 341:463-476

5. Jost RG, Quilligan EJ, Yeh SY, Anderson GC 1972 Intrauterine electroencephalogram of the sheep fetus. Am J Obstet Gynecol 114:535-539

6. Szeto HH 1983 Effects of narcotic drugs on fetal behavior activity: acute methadone exposure. Am J Obstet Gynecol 146:211-217

7. Blackman RB, Tukey JW 1958 The Measurement of Power Spectra. Dover Publications Inc, New York

8. Bloomfield P 1976 Fourier Analysis of Time Series: An Introduction. John Wiley and Sons, New York

9. Daniel WB 1978 Biostatistics: A Foundation for Analysis in the Health Sciences, 2nd ed. John Wiley and Sons, New York

10. Snedecor GW, Cochran WG 1967 Statistical Methods, 6th ed. The lowa State University Press, Ames, pp 130-132

11. Szeto $\mathrm{HH} 1990$ Spectral edge frequency as a simple quantitative measure of the maturation of electrocortical activity. Pediatr Res 27:289-292

12. Szeto HH, Hinman DJ 1985 Prenatal development of sleep-wake patterns in sheep. Sleep 8(4):347-355 